\title{
On the stability of intermetallic phases
}

\author{
S LELE, B N SARMA, A GHOSAL and G V S SASTRY \\ Centre of Advanced Study, Department of Metallurgical Engineering, Banaras Hindu Uni- \\ versity, Varanasi 221005 , India
}

\begin{abstract}
A general methodology using atomic clusters is applied to three problems connected to the study of alloy phase stability. The cluster method proposed by Allen and Cahn is applied to non-ideal hep structures under tetrahedral approximation using multiatom interactions. The possible ground-state structures which are stable at absolute zero temperature are obtained. A geometrical representation in $4 \mathrm{D}$ parameter space of the possible strengths of multiatom interactions permitted for these structures is illustrated in terms of a $2 \mathrm{D}$ analogue. Extending these ideas, the cluster variation method (CVM) proposed by Kikuchi is applied to fcc structures under tetrahedral approximation to find the effect of multiatom interactions on the topology of the coherent phase diagrams in which all the phases present are derivable by mere rearrangement of atoms on the parent disordered structure. In addition, the possible invariant reactions are identified in such coherent phase diagrams. Finally the CVM is applied for calculating a model incoherent phase diagram, that of $\mathrm{Ti}-\mathrm{Zr}$ system, where disordered hcp and bcc phases are present. The free energies of hcp and bcc phases are formulated using CVM procedures respectively under tetrahedral-octahedral and tetrahedral approximations. The CVM is shown to be in better agreement with the thermodynamic data and to be able to reproduce the correct value of measured enthalpy of transformation compared to that given by the regular solution model, which significantly overestimates the same.
\end{abstract}

Keywords. Phase stability; cluster method; cluster variation method; ground-state structures; coherent phase diagrams; incoherent phase diagrams.

\section{Introduction}

The stability of intermetallic phases is a topic of long-standing and continuing interest. Recently developed first-principles calculations are still not generally capable of predicting stability to within the limits of experimental errors. One has therefore to resort to physically acceptable models which use suitable empirical inputs and are mathematically tractable. This is usually accomplished by a general methodology which uses atomic clusters of finite size. A complete configurational description of a system containing $N$ atoms requires a representation in $N$-dimensional hyperspace, which is mathematically intractable. This problem can be simplified and the description can be made meaningful when atomic clusters are considered. The state of the order of the alloy can be accurately described (up to the size of the largest cluster considered) in terms of a much smaller number of variables: the probabilities of occurrence of each of the possible cluster configurations. As local order of the alloy can be described accurately in terms of the known atomic configurations of the clusters and their respective numbers, such descriptions can be made increasingly realistic by a hierarchy of cluster approximations. However, this has to be kept in balance with a very rapidly growing demand on computational resources as the cluster size increases. This is also a very realistic approach in the sense that frequently, the available experimental data are somewhat inaccurate and highly limited. Two promising methods which utilize the above ideas with remarkable success are the cluster method and the cluster variation method (CVM). Of these, the cluster method due to Allen and Cahn (1972) gives the so-called 
ground-state structures. These structures are stable at absolute zero temperature and are characterized by minimum configurational energies. On the other hand, CVM proposed by Kikuchi (1951) provides a systematic method of approximating the configurational entropy of a structure so that its finite temperature phase stability can be studied. CVM has been applied for the calculation of several phase diagrams ever since van Baal (1973) demonstrated the procedure for calculation of coherent fcc phase diagrams under tetrahedral approximation considering the effects of tetrahedral multiatom interactions. These methods together provide a consistent framework for studying the phase stability of alloys.

In this contribution, three applications which illustrate the versatility of this general approach are presented. They are: (i) determination of hcp ground states using cluster method under tetrahedral approximation considering 4-body interactions ( $\$ 2$ ), (ii) calculation of global phase diagrams for fcc ordering systems (coherent phase diagrams) using cluster variation method $(\S 3)$, and (iii) calculation of incoherent phase diagrams with coexisting disordered hcp and bcc phases respectively under tetrahedral-octahedral and tetrahedral approximations of CVM considering up to isotropic second-neighbour pair interactions ( $\$ 4)$.

\section{Ground-state structures}

In the cluster method proposed by Allen and Cahn (1972), the configurational energy of mixing of the alloy is expressed as a bilinear function of the cluster probabilities (state variables) and their energy coefficients. This configurational energy is then minimized in the presence of chosen clusters using the linear programming method, subject to certain constraint conditions on the cluster variables. The minimization conditions thus obtained define the requirements for the occurrence of the respective ground-state structures formed by the clusters chosen earlier. In this section, we obtain the ground-state structures of binary hcp alloys having non-ideal axial ratios using cluster method under tetrahedral approximation, considering 4-body tetrahedral multiatom interaction effects.

\subsection{Cluster variables and correlation functions}

In this method, at first a basic motif is chosen. The motif is a closed graph connecting a set of atomic sites in the structure under consideration. The motif sites are then populated by the atomic species $A$ and $B$ to obtain all the possible configurations of atomic clusters and subclusters. The probabilities of occurrence of these configurations are the corresponding cluster variables. However, all these variables are not independent. A convenient way of obtaining the independent set of variables, which governs the dimensionality of the ordering field, is through correlation functions. This is illustrated below.

Consider a crystal with $N$ atomic sites. In a binary (A-B) alloy, A atoms occupy $(1-C) N$ of these $N$ sites while the remaining $C N$ sites are occupied by $\mathrm{B}$ atoms. The site occupation operators are defined (Flinn 1956; Clapp and Moss 1966) through $p_{\mathrm{A}}^{\mathrm{i}}=1\left(p_{\mathrm{B}}^{\mathrm{i}}=1\right)$ if an $\mathrm{A}(\mathrm{B})$ atom occupies site $\mathrm{i}$ and $p_{\mathrm{A}}^{\mathrm{i}}\left(p_{\mathrm{B}}^{\mathrm{i}}\right)$ is zero otherwise. As vacancies are not considered,

$$
p_{\mathrm{A}}^{\mathrm{i}}+p_{\mathrm{B}}^{\mathrm{i}}=1 \text {. }
$$


Now, site operators $\sigma_{\mathrm{i}}$ are defined through

$$
\sigma_{\mathrm{i}}=\tau_{\mathrm{A}} p_{\mathrm{A}}^{\mathrm{i}}+\tau_{\mathrm{B}} p_{\mathrm{B}}^{\mathrm{i}}
$$

where $\tau_{A}$ and $\tau_{B}\left(\neq \tau_{A}\right)$ are arbitrary numbers. Averages of $\sigma_{i}$ or its products over crystallographically equivalent sites or clusters of sites are defined as the corresponding correlation functions. We have chosen $\tau_{\mathrm{A}}=0$ and $\tau_{\mathrm{B}}=1$ so that $\sigma_{\mathrm{i}}=p_{\mathrm{B}}^{\mathrm{i}}$ and correlation functions take the form $\left\langle p_{\mathrm{B}}^{\mathrm{i}}\right\rangle,\left\langle p_{\mathrm{B}}^{\mathrm{i}} p_{\mathrm{B}}^{\mathrm{j}}\right\rangle$, etc. The averages of the operators in (1) over all the sites are

$$
\left\langle p_{\mathrm{A}}^{\mathrm{i}}\right\rangle=\left\langle 1-p_{\mathrm{B}}^{\mathrm{i}}\right\rangle=1-\left\langle p_{\mathrm{B}}^{\mathrm{i}}\right\rangle=1-C \text { and }\left\langle p_{\mathrm{B}}^{\mathrm{i}}\right\rangle=C .
$$

The pair cluster variables are defined by

$$
y_{0}=\left\langle p_{\mathrm{A}}^{\mathrm{i}} p_{\mathrm{A}}^{\mathrm{j}}\right\rangle ; \quad y_{1}=\left\langle p_{\mathrm{A}}^{\mathrm{i}} p_{\mathrm{B}}^{\mathrm{j}}\right\rangle+\left\langle p_{\mathrm{B}}^{\mathrm{i}} p_{\mathrm{A}}^{\mathrm{j}}\right\rangle \quad \text { and } \quad y_{2}=\left\langle p_{\mathrm{B}}^{\mathrm{i}} p_{\mathrm{B}}^{\mathrm{j}}\right\rangle
$$

where $y_{0}, y_{1}$ and $y_{2}$ represent the probabilities of occurrence of atom pairs $\mathrm{AA}, \mathrm{AB}$ or $\mathrm{BA}$, and $\mathrm{BB}$ respectively on the pair of sites $\mathrm{i}$ and $\mathrm{j}$. In view of (1), $y_{0}$ and $y_{1}$ can be expressed in terms of $C$ and $y_{2}$ as follows:

$$
\begin{aligned}
& y_{0}=\left\langle\left(1-p_{\mathrm{B}}^{\mathrm{i}}\right)\left(1-p_{\mathrm{B}}^{\mathrm{j}}\right)\right\rangle=1-\left\langle p_{\mathrm{B}}^{\mathrm{i}}\right\rangle-\left\langle p_{\mathrm{B}}^{\mathrm{j}}\right\rangle+\left\langle p_{\mathrm{B}}^{\mathrm{i}} p_{\mathrm{B}}^{\mathrm{j}}\right\rangle=1-2 C+y_{2}, \\
& y_{1}=\left\langle\left(1-p_{\mathrm{B}}^{\mathrm{i}}\right) p_{\mathrm{B}}^{\mathrm{j}}\right\rangle+\left\langle p_{\mathrm{B}}^{\mathrm{i}}\left(1-p_{\mathrm{B}}^{\mathrm{j}}\right)\right\rangle=2\left\langle p_{\mathrm{B}}^{\mathrm{i}}\right\rangle-2\left\langle p_{\mathrm{B}}^{\mathrm{i}} p_{\mathrm{B}}^{\mathrm{j}}\right\rangle=2\left(C-y_{2}\right)
\end{aligned}
$$

Thus, in an alloy of fixed composition, only one pair variable remains independent. It follows from the above that the cluster variables can all be expressed as linear combinations of a set of independent correlation functions. This set contains one correlation function corresponding to each of the distinct subgraphs contained in the motif and the motif itself. (For the sake of convenience, we shall use $\langle p i j\rangle$ to represent the correlation function $\left\langle p_{\mathrm{B}}^{\mathrm{i}} p_{\mathrm{B}}^{\mathrm{j}}\right\rangle$ in the following.)

\subsection{Configurational energy}

For the study of ground states in this section, we choose a distorted tetrahedron connecting four adjacent sites in a non-ideal hcp structure as shown by vertices 1-2-3-4 in figure 1. Eight distinct types of clusters can occur. The number of equivalent clusters of each type (statistical weights $w_{i}$ ) are identified by rotational symmetry considerations. The details of these clusters are depicted in table 1. By extending the argument of correlation functions in $\$ 2.1$ to the tetrahedral correlations, we find that only five correlation functions, namely $\langle p 12\rangle,\langle p 14\rangle,\langle p 123\rangle$, $\langle p 124\rangle$ and $\langle p 1234\rangle$ are independent when the composition is fixed, suggesting that three constraint conditions should be operating on $Z_{\mathrm{i}}$. They are obtained as explained in the following. We express $Z_{\mathrm{i}}$ in terms of the above five correlation functions by a procedure similar to that used for obtaining (4). Elimination of the correlation functions from this set of equations yields the following constraints:

$$
\begin{aligned}
& Z_{0}+Z_{1}+Z_{2}+Z_{3}+Z_{4}+Z_{5}+Z_{6}+Z_{7}=1, \\
& Z_{1}+Z_{2}+2 Z_{3}+2 Z_{4}+3 Z_{5}+3 Z_{6}+4 Z_{7}=4 C, \\
& Z_{1}-3 Z_{2}+2 Z_{3}-2 Z_{4}+3 Z_{5}-Z_{6}=0 .
\end{aligned}
$$




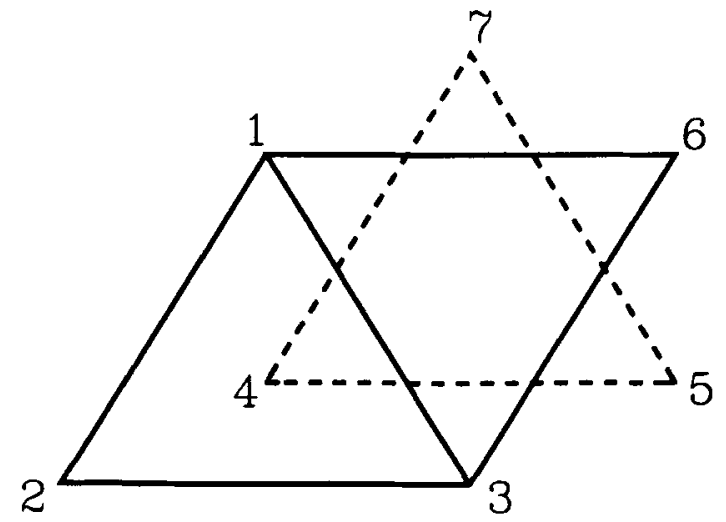

Figure 1. Projection of tetrahedral-octahedral motif on the close-packed plane in hep or fcc structures. Sites 1-2-3-4 join to form a tetrahedron whereas sites 1-3-4-5-6-7 form the vertices of an octahedron. Continuous lines join sites on one layer and broken lines join sites on an adjacent one.

Table 1. Tetrahedral cluster configurations and their energies.

\begin{tabular}{lcccccc}
\hline $\begin{array}{l}\text { Cluster } \\
\text { type } \\
\mathrm{i}\end{array}$ & $\begin{array}{c}\text { Cluster } \\
\text { probability } \\
Z_{\mathrm{i}}\end{array}$ & $\begin{array}{c}\text { Cluster } \\
\text { configuration } \\
\text { (sites occupied } \\
\text { by B atoms) }\end{array}$ & $\begin{array}{c}\text { Weight } \\
\text { of the } \\
\text { cluster } \\
w_{\mathrm{i}}\end{array}$ & $\begin{array}{c}\text { Concen- } \\
\text { tration of } \\
\text { the cluster } \\
c_{\mathrm{i}}\end{array}$ & $\begin{array}{c}\text { Number of first } \\
\text { and second neighbour } \\
\text { unlike pairs } \\
n_{\mathrm{i}}^{\mathrm{I}}+n_{\mathrm{i}}\end{array}$ & $\begin{array}{c}\text { Energy } \\
\text { coefficient } \\
\text { of cluster } \\
\text { variable }\end{array}$ \\
\hline 0 & $Z_{0}$ & - & 1 & 0 & 0 & 0 \\
1 & $Z_{1}$ & 1 & 3 & $1 / 4$ & 3 & $3 / 2 W(1+\alpha)$ \\
2 & $Z_{2}$ & 4 & 1 & $1 / 4$ & 3 & $3 / 2 W(1+\beta)$ \\
3 & $Z_{3}$ & 1,2 & 3 & $1 / 2$ & 4 & $2 W$ \\
4 & $Z_{4}$ & 1,4 & 3 & $1 / 2$ & 4 & $2 W$ \\
5 & $Z_{5}$ & $1,2,3$ & 1 & $3 / 4$ & 3 & $3 / 2 W(1+\gamma)$ \\
6 & $Z_{6}$ & $1,2,4$ & 3 & $3 / 4$ & 3 & $3 / 2 W(1+\delta)$ \\
7 & $Z_{7}$ & $1,2,3,4$ & 1 & 1 & 0 & 0 \\
\hline
\end{tabular}

The configurational energy $E$ of the alloy can be expressed in terms of cluster variables and their corresponding energies as

$$
E=2 N \sum_{\mathrm{i}=0}^{7} Z_{\mathrm{i}} \varepsilon_{\mathrm{i}}
$$

Here $2 N$ is the number of tetrahedra arising from $N$ atomic sites. The configurational energy of mixing $U$ can be found by subtracting from $E$ the energy of a mechanical mixture of $A$ and $B$ having the same composition. By utilizing the constraint conditions (5)-(7), $U$ can be expressed as follows:

$$
\begin{aligned}
U / N= & 2\left[Z_{1}\left(\varepsilon_{1}-1 / 2 \varepsilon_{0}-1 / 4 \varepsilon_{3}+1 / 4 \varepsilon_{4}\right)+Z_{2}\left(\varepsilon_{2}-3 / 2 \varepsilon_{0}+3 / 4 \varepsilon_{3}-3 / 4 \varepsilon_{4}-\varepsilon_{7}\right)\right. \\
& +Z_{3}\left(1 / 2 \varepsilon_{3}+1 / 2 \varepsilon_{4}-1 / 2 \varepsilon_{0}-1 / 2 \varepsilon_{7}\right)+Z_{4}\left(1 / 2 \varepsilon_{3}+1 / 2 \varepsilon_{4}-1 / 2 \varepsilon_{0}-1 / 2 \varepsilon_{7}\right) \\
& \left.+Z_{5}\left(\varepsilon_{5}+1 / 2 \varepsilon_{0}-3 / 4 \varepsilon_{3}+3 / 4 \varepsilon_{4}\right)+Z_{6}\left(\varepsilon_{6}-1 / 2 \varepsilon_{0}+1 / 4 \varepsilon_{3}-1 / 4 \varepsilon_{4}-\varepsilon_{7}\right)\right] .
\end{aligned}
$$


We note that only five independent combinations of the eight cluster energies, $\varepsilon_{i}$, appear in $U$.

A convenient and physically meaningful abbreviated notation for these energy combinations can be chosen in terms of an effective pair interchange energy $W$, and four so-called multiatom interaction parameters $\alpha, \beta, \gamma$ and $\delta$ as given in table 1 . These energies reduce to those given by the pairwise-interactions model when the axial ratio becomes ideal and multiatom interaction parameters vanish.

\subsection{Linear programming method}

To obtain ground state in the presence of a triplet of clusters, we solve for three corresponding cluster variables from (5)-(7) and eliminate them from the energy expression in (9). If the energy coefficients of $Z_{i}$ present in this equation are all positive, then $U$ will be minimum when the $Z_{\mathrm{i}}$ appearing in the equation themselves vanish. The requirement that the energy coefficients of $Z_{\mathrm{i}}$ should be positive defines the ranges of multiatom interaction parameters permissible for the occurrence of ground states. In the case of cluster triplet $\left(Z_{0}, Z_{1}, Z_{2}\right)$, when the ordering tendency is considered for which $W<0$, if

$$
\begin{aligned}
& -1-3 \alpha<0 ; \quad-2-3 \alpha-3 \beta<0 ; \quad-2-3 \alpha+\gamma<0 \\
& -2-2 \alpha-\beta+\delta<0 ; \quad-4-3 \alpha-\beta<0
\end{aligned}
$$

then the minimum in $U$ occurs at

$$
\begin{aligned}
U / N & =3 C W(4+3 \alpha+\beta), \\
Z_{3} & =Z_{4}=Z_{5}=Z_{6}=Z_{7}=0, \\
Z_{0} & =1-4 C, Z_{1}=3 C, Z_{2}=C .
\end{aligned}
$$

As $0 \leqslant Z_{i} \leqslant 1$, we get $0 \leqslant C \leqslant 0 \cdot 25$.

At $C=0$, pure $A$ is stable whereas at the other end of this composition range, at $C=0 \cdot 25$, two distinct degenerate superstructures $A_{3} B^{1}$ (Strukturbericht type $\mathrm{DO}_{19}$ ) and $A_{3} B^{3}$ are stabilized with equal but minimum energies. [Our nomenclature for superstructures follows that used by Singh et al (1991).] These structures which are stable at the ends of composition ranges are known as terminal superstructures. At all the intermediate values of the composition range, phase mixtures of these terminal superstructures are stable. In principle, an infinite number of degenerate intermediate superstructures are also possible. This procedure is repeated for all $\left(\begin{array}{l}8 \\ 3\end{array}\right)$ possible cluster triplets to complete the search for ground-state structures.

The linear programming method ensures a minimum in energy whenever a solution can be found in terms of the cluster triplets from the conditions (5)-(7). In this case, for eight of the cluster triplets exemplified by $\left(Z_{0}, Z_{1}, Z_{3}\right)$ there are no solutions in terms of other clusters. Similarly, for triplets of type $\left(Z_{0}, Z_{\mathrm{i}}, Z_{7} ; \mathrm{i}=1-6\right)$, it is not possible to make the cluster coefficients simultaneously positive when $W<0$. Further, this method cannot decide whether the superstructures made up of more than three clusters belong to ground state. 


\subsection{Parameter space for ground-state structures}

We shall illustrate the representation of permitted domain of the parameter space for the occurrence of ground state structures. The parameter space is a $4 \mathrm{D}$ hyperspace spanned by the multiatom interaction parameters $\alpha, \beta, \gamma$ and $\delta$. For the cluster triplet $\left(Z_{0}, Z_{1}, Z_{2}\right)$ we have five inequalities in (10) each of which defines a half-space in 4D hyperspace bounded by an infinitely extended $3 \mathrm{D}$ hyperplane represented by the corresponding equality. The intersection of these half-spaces defines the permitted domain of parameter space. The procedure for obtaining the permitted domain is illustrated in the following for a $2 \mathrm{D}$ case. We begin with three inequalities defining half-spaces in 2D where the corresponding straight lines are non-parallel. As shown in figure 2, the permitted domain can then be obtained in the following manner. Generally, the lines intersect in pairs giving rise to three vertices. Each of the vertices lies in the permitted domain only when it lies in the half-space on the permitted side of the third line; otherwise the vertex is in a non-permitted domain. Four cases can arise:

(i) When all the three points are permitted, as can be seen from figure $2 \mathrm{a}$, the permitted domain $P$ is a $2 \mathrm{D}$ simplex, the triangle.

(ii) When two of the three points are permitted, the permitted domain is a union of the line segment joining the permitted points and an angle whose edges are

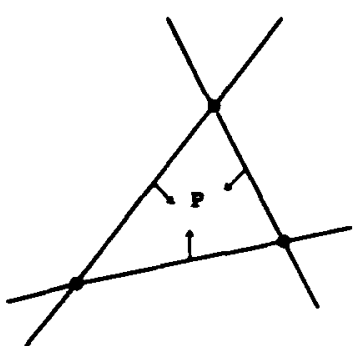

(a)

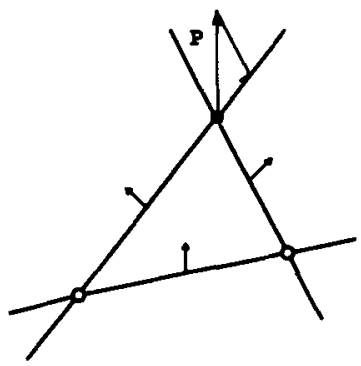

(c)

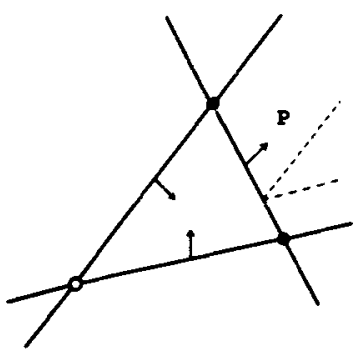

(b)

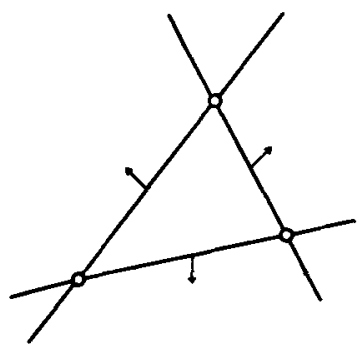

(d)

Figare 2. Graphical representation of the possible cases of permitted domains $(P)$ in $2 D$ when three inequalities are present, as explained in section 2.4. Arrows on one side of the lines indicate the half-space permitted. Filled (open) circles represent permitted (non-permitted) vertices. 
parallel to the rays originating at the permitted vertices along the directed segments from the non-permitted vertex through permitted vertices.

(iii) When only one of the three vertices is permitted, then $P$ is an angle (pyramidal cone for higher-dimensional case) with its vertex at the permitted point and edges originating at the vertex along the directed segments from the nonpermitted points through the permitted vertex. Then any point in $P$ can be spanned by moving appropriate lengths along the edges of the angle, as suggested in figure $2 \mathrm{c}$.

(iv) When none of the three points is permitted, there is no permitted domain (figure 2d).

An extension of this procedure was utilized for determining the permitted domains for each of the ground-state structures by Sarma et al (1994). This gives an idea of the possible strengths of the 4-body multiatom interaction effects in a binary alloy system depending on the actual structures present. The results of the ground-state analysis are presented in table 2.

\section{Coherent fcc ordering phase diagrams}

Coherent phase diagrams are those in which all the phases present are derivable from the disordered parent lattice by mere rearrangement of atomic species. CVM has been applied to fcc alloys undergoing order-disorder transformations under tetrahedral approximation to investigate the effects of 4-body tetrahedral interactions on the topology of the possible phase diagrams. Global behaviour of such systems has been investigated and the possible invariant reactions have been identified.

\subsection{Cluster variation and natural iteration methods}

Due to the preferential occupancy of certain sets of sites by some atomic species during ordering reactions, one has to divide the atomic sites into a suitable number of sublattices for a complete description of the system. The fcc structure is here subdivided into four interpenetrating simple cubic sublattices, denoted by $a, b, c$ and $d$ to treat $L 1_{2}$ and $L 1_{0}$

Table 2. Summary of ground-state superstructures.

\begin{tabular}{|c|c|c|}
\hline Superstructures & Cluster concentration & Prototypes \\
\hline \multicolumn{3}{|c|}{ Terminal superstructures } \\
\hline $\begin{array}{l}\mathrm{A}_{3} \mathrm{~B}^{1}\left(\mathrm{DO}_{19}\right) / \mathrm{A}_{3} \mathrm{~B}^{3} \\
\text { (degenerate) }\end{array}$ & $Z_{1}=3 Z_{2}=3 / 4$ & $\begin{array}{c}\mathrm{Ti}_{3} \mathrm{Al}, \mathrm{Ni}_{3} \mathrm{Sn}, \\
\mathrm{Mg}_{3} \mathrm{Cd} \text {, etc. }\end{array}$ \\
\hline $\mathbf{A}_{2} \mathbf{B}^{2}$ & $Z_{1}=2 Z_{4}=2 / 3$ & $\mathrm{Pt}_{2} \mathrm{Ta}$ \\
\hline $\mathbf{A B}^{1}(\mathbf{B h})$ & $Z_{2}=Z_{5}=1 / 2$ & LiRh \\
\hline $\begin{array}{l}\mathbf{A B}^{2}(\mathbf{B} 19) / \mathbf{A B}^{4} \\
\text { (degenerate) }\end{array}$ & $Z_{3}=Z_{4}=1 / 2$ & MgCd \\
\hline $\begin{array}{l}\mathrm{AB}^{3} / \mathrm{AB}^{5} \\
\text { (degenerate) }\end{array}$ & $Z_{1}=Z_{6}=1 / 2$ & $\cdots$ \\
\hline \multicolumn{3}{|c|}{ Intermediate superstructures } \\
\hline $\begin{array}{l}A_{5} B \\
A_{4}^{B} B \\
A_{1 n} B_{3}\end{array}$ & $\begin{array}{l}Z_{0}=1 / 3, Z_{1}=3 Z_{2}=1 / 2 \\
Z_{0}=1 / 3, Z_{1}=Z_{2}=1 / 5 \\
Z_{0}=1 / 13, Z_{1}=9 / 13, Z_{2}=3 / 13\end{array}$ & $\begin{array}{c}\mathrm{Al}_{5} \mathrm{~W} \\
\mathrm{Au}_{4} \mathrm{Zr} \\
\mathrm{Cu}_{10} \mathrm{Sb}_{3}\end{array}$ \\
\hline
\end{tabular}


types of ordering. Point variables and pair variables for each sublattice are defined according to equations similar to those in (1)-(4). Atoms are permuted on the sites of a regular tetrahedral motif so as to obtain the distinct tetrahedral cluster configurations and the corresponding variables. There are five tetrahedral cluster variables $Z_{i}$ $(i=0$ to 4 ) in the case of a disordered alloy and the configurational energy of mixing in this case can be expressed as first shown by van Baal (1973) as follows:

$$
U / N=3 W(1+\alpha) Z_{1}+4 W Z_{2}+3 W(1+\beta) Z_{3},
$$

where $\alpha$ and $\beta$ are the tetrahedral multiatom iteration parameters. Note the similarity with (9).

Then the configurational entropy of the alloy is calculated by CVM (Kikuchi 1951). In this method, the largest $r$-point clusters considered are assumed to be randomly distributed on the set of atomic sites and the Boltzmann statistical entropy summations are obtained. In doing so, it is required that all the cluster and subcluster configurations are counted only once. The configurational entropy in tetrahedral approximation is given by (van Baal 1973)

$$
S=s_{4}-s_{2}+5 s_{1},
$$

where $s_{r}$ is the Boltzmann statistical entropy summation for $r$-point clusters. As $U$ and $S$ are expressed as functions of $Z_{i}$, the equilibrium distribution of $Z_{i}$ is found by minimizing the Helmholtz free energy

$$
F=U-T S,
$$

with respect to each of the independent cluster variables by keeping the composition fixed. Since for condensed phases the difference between $F$ and the Gibbs free energy $G$ is very small, we shall not distinguish the two. An alternative method of arriving at equilibrium is through minimization of grand potential $\Omega$ defined through

$$
\Omega=G-(1-C) \mu_{\mathrm{A}}-C \mu_{\mathrm{B}},
$$

where $\mu_{\mathbf{A}}$ and $\mu_{\mathbf{B}}$ are chemical potentials of atomic species $\mathbf{A}$ and $\mathbf{B}$. The conditions of equilibrium are a set of nonlinear equations, solvable for $Z_{\mathrm{i}}$ either by NewtonRaphson method or by so-called natural iteration method (NIM), especially developed for this purpose by Kikuchi (1974). In this part of the work NIM was used for obtaining equilibrium values of $Z_{i}$. The conditions of equilibrium in this case are obtained as

$$
Z_{\mathrm{i}}=\exp \left[\frac{\Omega}{2 R T}-\frac{1}{R T}\left(\varepsilon_{\mathrm{i}}-\frac{\left(\mu_{m}+\mu_{n}+\mu_{p}+\mu_{q}\right)}{8}\right)\right] Y^{1 / 2} X^{-5 / 8},
$$

where $\varepsilon_{\mathrm{i}}$ are the energy coefficients of $Z_{\mathrm{i}}$ as appearing in $U(12) ; \mu_{m}, \mu_{n}, \mu_{p}, \mu_{q}$ are equal to $\mu_{\mathrm{A}}$ or $\mu_{\mathrm{B}}$ depending on the occupancy of the sites $m, n, p, q$ in the tetrahedral cluster by $A$ or $B$ atoms respectively; and $X$ and $Y$ are respectively the products of all point and pair variables.

The above equations are solved iteratively to yield values of $\Omega$ and $Z_{\mathrm{i}}$ for selected values of $\mu=\left(\mu_{\mathrm{B}}-\mu_{\mathrm{A}}\right) / 2$ and $T$. Equilibrium between a pair of phases occurs when each of $\Omega$ and $\mu$ are equal for both the phases. The corresponding compositions can be found from the $Z_{\mathrm{i}}$ evaluated earlier. 


\subsection{Effect of multiatom interactions on the topology of phase diagrams}

An analysis of ground-state structures of fcc alloys under tetrahedral approximation of cluster method using multiatom interactions by Cahn and Kikuchi (1979) showed that for ordering systems where $W<0$, the phases $\mathrm{A}_{3} \mathrm{~B}, \mathrm{AB}$ and $\mathrm{AB}_{3}$ are stable within a triangular domain (XYZ in figure 3 ) of the parameter space spanned by $\alpha$ and $\beta$. In order to investigate the effects of multiatom interactions on the topology of typical fcc ordering phase diagrams involving three ordered phases, a symmetric half of the permitted domain (triangle PQR in figure 3) was chosen and phase diagrams were calculated using values of $\alpha$ and $\beta$ within this domain. The invariant reactions were identified in each case. Results are summarized in table 3 . As can be seen from figure 3, equal values of $\alpha$ and $\beta$ gave rise to symmetric phase diagrams. At $P, A B$ is stabilized compared to $A_{3} B$ and $A_{3}$ with the presence of peritectoid reactions. At $Q$, there is an exactly opposite tendency and the reaction is eutectoid type $\mathrm{I}$. At $\mathrm{R}, \mathrm{A}_{3} \mathrm{~B}$ phase is very strongly stabilized and for compositions below $50 \%$, eutectoid type II reaction takes place in which an ordered phase transforms to a disordered one together with another ordered phase. For a detailed analysis of the results, reference can be made to Ghosh-Moulic et al (1994). This investigation thus gives a clear idea of the various possible effects of the multiatom interactions on the phase stability of fec alloys.
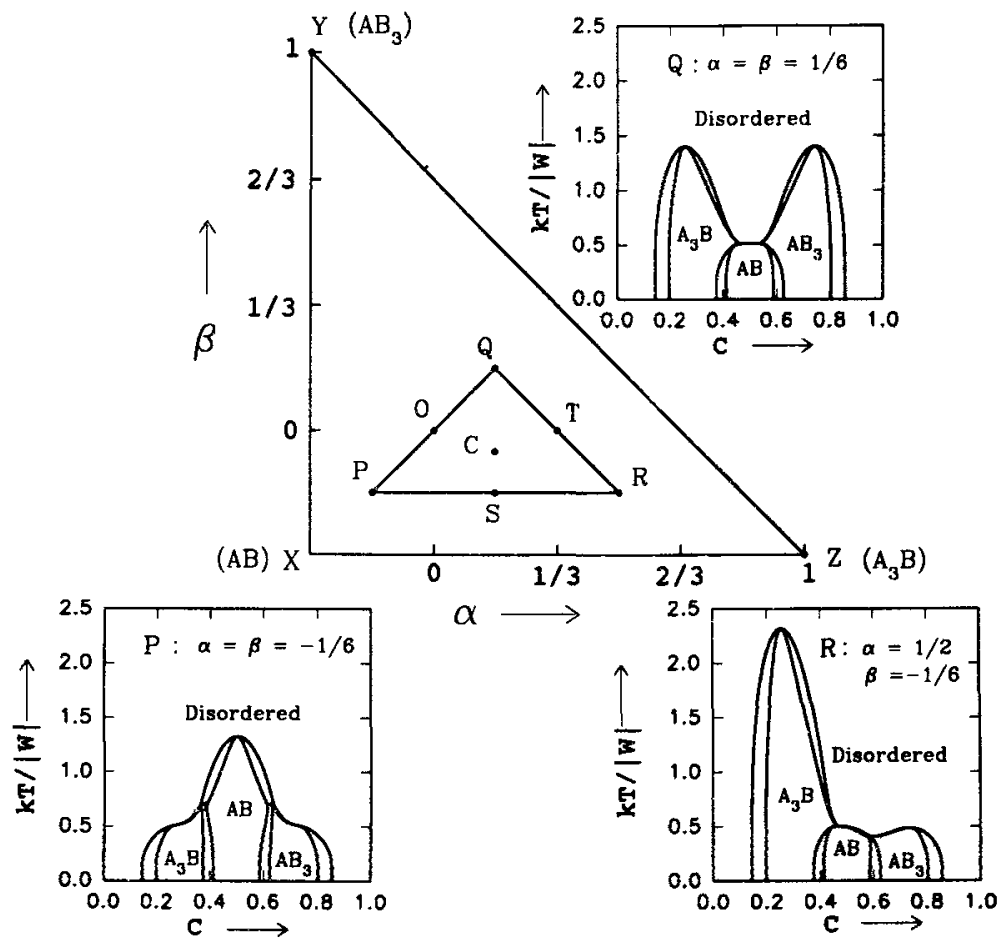

Figure 3. Permitted domain of the parameter space $(\alpha, \beta)$ for the stability of $\mathrm{L}_{2}$ and $\mathrm{L1}_{0}$ structures. Typical phase diagrams obtainable for different choices of multiatom interaction parameters $\alpha$ and $\beta$ are also shown. 
Table 3. Nature of invariant reactions for various values of multiatom interaction parameters $\alpha$ and $\beta$.

\begin{tabular}{|c|c|c|c|c|}
\hline \multirow{2}{*}{$\begin{array}{l}\text { Points in } \\
\text { parameter } \\
\text { space }\end{array}$} & \multirow{2}{*}{$\alpha$} & \multirow{2}{*}{$\beta$} & \multicolumn{2}{|c|}{ Invariant reaction } \\
\hline & & & $C<0.5$ & $C>0.5$ \\
\hline 0 & 0 & 0 & Eutectoid I & Eutectoid I \\
\hline $\mathbf{P}$ & $-1 / 6$ & $-1 / 6$ & Peritectoid & Peritectoid \\
\hline $\mathbf{Q}$ & $1 / 6$ & $1 / 6$ & Eutectoid I & Eutectoid I \\
\hline $\mathbf{R}$ & $1 / 2$ & $-1 / 6$ & Eutectoid II & Peritectoid \\
\hline $\mathbf{S}$ & $1 / 6$ & $-1 / 6$ & Eutectoid I & Peritectoid \\
\hline $\mathbf{T}$ & $1 / 3$ & 0 & Eutectoid II & Eutectoid I \\
\hline C & $1 / 6$ & $-1 / 18$ & Eutectoid I & Peritectoid \\
\hline
\end{tabular}

Eutectoid I $:$ Disordered $\rightarrow \mathrm{AB}+\mathrm{A}_{3} \mathbf{B} / \mathbf{A B}_{3}$
Eutectoid II $: \mathbf{A}_{3} \mathrm{~B} \rightarrow$ Disordered $+\mathbf{A B}$
Peritectoid $:$ Disordered $+\mathrm{AB} \rightarrow \mathrm{A}_{3} \mathrm{~B} / \mathbf{A B}_{3}$

\section{Calculation of incoherent phase diagrams: $\mathrm{Ti}-\mathrm{Zr}$ system}

Incoherent phase diagrams are those in which the phases present belong to more than one parent disordered structure. An example of such systems is the $\mathrm{Ti}-\mathrm{Zr}$ system in which disordered hcp $\alpha$ phase transforms to disordered bcc $\beta$ phase. CVM has been applied for a calculation of phase diagram of $\mathrm{Ti}-\mathrm{Zr}$ system in the following.

The free energy of hcp $\alpha$ phase is calculated under tetrahedral-octahedral (TO) approximation of CVM. As shown in figure 1, a motif joining seven nearby sites in the disordered hcp structure is considered in which sites $1,2,3,4$ join a distorted tetrahedron (which is also used in $\S 2$ ), and sites $1,3,4,5,6,7$ join a distorted octahedron, the distortions being due to the nonideal axial ratio. There are a total of 13 correlation functions corresponding to this motif as governed by the space group symmetry of hcp structure. Among them, $\langle p 12\rangle,\langle p 14\rangle,\langle p 123\rangle,\langle p 124\rangle$ and $\langle p 1234\rangle$ arise due to tetrahedral clusters and the additional ones, viz. $\langle p 15\rangle,\langle p 136\rangle$, $\langle p 135\rangle,\langle p 1357\rangle,\langle p 1346\rangle,\langle p 1345\rangle,\langle p 13456\rangle$ and $\langle p 134567\rangle$, arise due to octahedral clusters.

The configurational energy of mixing $U$ can be obtained by a procedure similar to that given in $\S 2$.

$$
U / N=\left(\langle p 12\rangle-C^{\alpha}\right) 3 e_{12}^{\alpha}+\left(\langle p 14\rangle-C^{\alpha}\right) 3 e_{14}^{\alpha}+\left(\langle p 15\rangle-C^{\alpha}\right) 3 e_{15}^{\alpha} .
$$

By extending the CVM arguments as illustrated in $\S 3$ to the case of TO approximation, the configurational entropy $S_{\text {TO }}$ can be calculated as follows:

$$
S_{\mathrm{TO}}=s_{1234}+s_{134567}-s_{123}-s_{136}-s_{124}+s_{12}+s_{14}-s_{1} \text {, }
$$

where $s_{\mathrm{i}}$ represents the Boltzmann entropy summation for the cluster $\mathrm{i}$.

Then $G$ in (14) and the chemical potentials of $\alpha$ phase can be calculated. This $G$ can be minimized with respect to each of the $\langle p i\rangle$ in order to obtain the conditions for the internal equilibrium of $\alpha$ phase. Similar procedures are adopted for the bcc $\beta$ phase under tetrahedral approximation of CVM (Kikuchi and van Baal 1974; Kikuchi 1987) including interactions up to second-neighbour distances. If the hcp $\alpha$ phase is taken as 
the reference state, then the chemical potentials of the bcc $\beta$ phase are obtained from

Here,

$$
\begin{aligned}
& \mu_{\mathrm{A}}^{\beta}=\Delta G_{\mathrm{A}}^{\alpha \rightarrow \beta}+\left(G^{\beta}-C^{\beta} \frac{\mathrm{d} G^{\beta}}{\mathrm{d} C^{\beta}}\right) \\
& \mu_{\mathrm{B}}^{\beta}=\Delta G_{\mathrm{B}}^{\alpha \rightarrow \beta}+\left(G^{\beta}+\left(1-C^{\beta}\right) \frac{\mathrm{d} G^{\beta}}{\mathrm{d} C^{\beta}}\right) .
\end{aligned}
$$

$$
\Delta G_{\mathrm{A}}^{\alpha \rightarrow \beta}=\Delta H_{\mathrm{A}}^{\alpha \rightarrow \beta}\left(1-\frac{T}{T_{\mathrm{A}}^{\alpha \rightarrow \beta}}\right) \text { and } \Delta G_{\mathrm{B}}^{\alpha \rightarrow \beta}=\Delta H_{\mathrm{B}}^{\alpha \rightarrow \beta}\left(1-\frac{T}{T_{\mathrm{B}}^{\alpha \rightarrow \beta}}\right),
$$

are the transformation free energies of $\mathrm{A}$ and $\mathrm{B}$ respectively, $G^{\beta}$ the free energy, and $C^{\beta}$ the composition of the $\beta$ phase. For the case of $\mathrm{Ti}-\mathrm{Zr}$ system, the necessary thermodynamic data are taken from Hultgren et al (1973).

Thermodynamic equilibrium between both the phases requires equality of chemical potentials of each component in both the phases. Modified Newton's method is used to arrive at the energy values corresponding to equilibrium.

In the present calculation, we have chosen a set of four energy parameters corresponding to isotropic first-neighbour $\left(e_{12}^{\alpha}=e_{14}^{\alpha}\right)$ and second-neighbour $\left(e_{15}^{\alpha}\right)$ pair interactions in the $\alpha$ phase and first- $\left(e_{\mathrm{I}}^{\beta}\right)$ and second- $\left(e_{\mathrm{II}}^{\beta}\right)$ neighbour pair interactions in the $\beta$ phase which can reproduce the observed value of $\Delta H^{\alpha \rightarrow \beta}=2460 \mathrm{~J} \mathrm{~mol}^{-1}$ (Auffredic et al 1982) for the congruent transformation (temperature fixed at $878 \mathrm{~K}$ as suggested by Auffredic et al (1982) and Murray (1987)) and also give approximately the observed width of the two-phase field $(\alpha+\beta)$ at a temperature of $1013 \mathrm{~K}$. The calculated phase diagram is given in figure 4 .

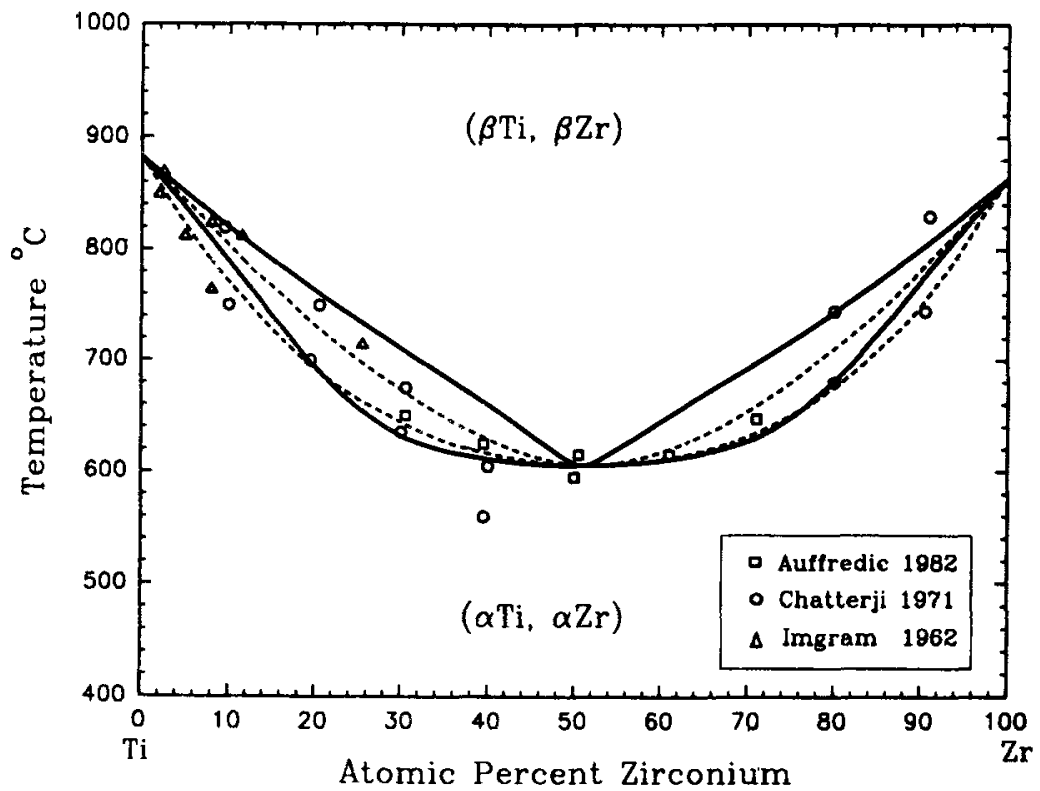

Figure 4. Phase diagram of $\mathrm{Ti}-\mathrm{Zr}$ system showing selected experimental data. Continuous lines show phase diagram calculated in the present work using CVM whereas broken lines show that from Murray (1987) using regular solution model. 
The set of energy parameters used for the calculation of this phase diagram are

$$
\begin{aligned}
& e_{12}^{\alpha}=e_{14}^{\alpha}=-2300 \mathrm{~J} \mathrm{~mol}^{-1}, \\
& e_{15}^{\alpha}=-500 \mathrm{~J} \mathrm{~mol}^{-1} \\
& e_{\mathrm{I}}^{\beta}=-1038 \mathrm{~J} \mathrm{~mol}^{-1} \\
& e_{\mathrm{II}}^{\beta}=-5623 \mathrm{~J} \mathrm{~mol}^{-1}
\end{aligned}
$$

An earlier attempt by Murray (1987) using regular solution model does not reproduce the observed (Auffredic et al 1982) value of $\Delta H^{\alpha \rightarrow \beta}$ for equiatomic alloys. The regular solution model estimate for this is $3197 \mathrm{~J} \mathrm{~mol}^{-1}$ and the overestimation is well beyond experimental error.

The phase diagram obtained using regular solution model by Murray (1987) is also shown in figure 4 along with selected experimental data. The $(\alpha+\beta)$ phase field found by Murray is much narrower than that suggested by the experimental data. The present calculations are in better agreement with the data in this respect. No attempt has been made, however, to optimize the energy parameters and the phase diagram. Also, multiatom interactions have been neglected. The main objective has been to demonstrate the capability of the CVM procedures to simultaneously represent thermodynamic and phase diagram data in an adequate manner. Availability of additional and more accurate data would make the modelling more realistic.

\title{
5. Conclusions
}

We have considered three applications of a general methodology using atomic clusters each of which was designed to take us closer to solution of problems which are of much practical interest. These procedures can be extended to the study of phase stability in more complex systems such as $\mathrm{Ti}-\mathrm{Al}-\mathrm{Nb}$. This involves all the three common metallic structures in disordered and/or ordered forms and presents greater computational difficulties. In addition, interfacial energies for stacking faults and anti-phase boundaries can be obtained as part of the calculation.

\section{Acknowledgements}

This paper is based on an invited talk by the first author in an Indo-Japanese seminar on new materials which was supported by the Department of Science and Technology, India, and the Japan Society for Promotion of Science, Japan. This work was carried out as part of a research project funded by the Council of Scientific and Industrial Research, India.

\section{References}

\author{
Allen S M and Cahn J W 1972 Acta Metall. 20423 \\ Auffredic J P, Etchessahar E and Debuigne 1982 J. Less-Common Metals 8449 \\ Cahn J W and Kikuchi R 1979 Acta Metall. 271329 \\ Clapp P C and Moss S C 1966 Phys. Rev. 142418 \\ Flinn P A 1956 Phys. Rev. 104350
}


Ghosh-Moulic A, Sastry G V S and Lele S 1994 Bull. Mater. Sci. 17439

Hultgren R, Desai P D, Hawkins D T, Gleiser M, Kelley K K and Wagman D D 1973 Selected values of the thermodynamic properties of the elements (Metals Park, OH: ASM) p. 516, p. 575

Kikuchi R 1951 Phys. Rev. 81988

Kikuchi R 1974 J. Chem. Phys. 601071

Kikuchi R 1987 Physica A142 321

Kikuchi $R$ and van Baal C M 1974 Scr. Metall. 8425

Murray J L (ed.) 1987 in Phase diagrams of binary titanium alloys (Metals Park, OH.: ASM) p. 340

Sarma B N, Ravisankar N, Gopal M, Singh A K, Prasad R and Lele S 1994 Philos. Mag. B70 1117

Singh A K, Singh V and Lele S 1991 Acta Metall. Mater. 392847

van Baal C M 1973 Physica 64571 